\title{
Contagion on Networks with Self-Organised Community Structure
}

\author{
Alberto Antonioni ${ }^{1}$, Seth Bullock ${ }^{2}$, Christian Darabos ${ }^{3}$, Mario Giacobini ${ }^{4}$ \\ Bryan N. Iotti ${ }^{4}$, Jason H. Moore ${ }^{5}$, Marco Tomassini ${ }^{1}$ \\ ${ }^{1}$ Information Systems Department, Faculty of Business and Economics, University of Lausanne, Switzerland \\ ${ }^{2}$ Institute for Complex Systems Simulation, University of Southampton, United Kingdom \\ ${ }^{3}$ Computational Genetics Laboratory, Geisel School of Medicine, Dartmouth College, USA \\ ${ }^{4}$ Computational Epidemiology Group, Department of Veterinary Sciences, University of Torino, Italy \\ ${ }^{5}$ Institute for Biomedical Informatics, Perelman School of Medicine, University of Pennsylvania, USA
}

\begin{abstract}
Living systems are organised in space. This imposes constraints on both their structural form and, consequently, their dynamics. While artificial life research has demonstrated that embedding an adaptive system in space tends to have a significant impact on its behaviour, we do not yet have a full account of the relevance of spatiality to living self-organisation.

Here, we extend the REDS model of spatial networks with self-organised community structure to include the "small world" effect. We demonstrate that REDS networks can become small worlds with the introduction of a small amount of random rewiring. We then explore how this rewiring influences two simple dynamic processes representing the contagious spread of infection or information.

We show that epidemic outbreaks arise more easily and spread faster on REDS networks compared to standard random geometric graphs (RGGs). Outbreaks spread even faster on small world REDS networks (due to their shorter path lengths) but initially find it more difficult to establish themselves (due to their reduced community structure). Overall, we find that small world REDS networks, with their combination of short characteristic path length, positive assortativity, strong community structure and high clustering, are more susceptible to a range of contagion dynamics than RGGs, and that they offer a useful abstract model for studying dynamics on spatially organised living systems.
\end{abstract}

\section{Introduction}

The structure of living systems such as cells, brains, organisms, colonies and ecosystems is neither random, where all interactions between system components are equally likely, nor regular, where interactions between system elements are arranged uniformly. Rather, natural systems tend to exhibit complex structure, featuring clustering and modularity.

Despite this, many models of living systems either assume that the system is well-mixed, with every interaction between system components equally valid, or specify that the interactions within a system are embedded on a regular lattice. The behaviour of such models is sensi- tive to these choices regarding how interactions are structured, with, for instance, spatial embedding often encouraging complex organised behaviour where it would not otherwise arise (e.g., Boerlijst and Hogeweg, 1991; Di Paolo, 2000; Buckley et al., 2010).

To some extent, the complex structure of living systems arises as a consequence of adaptation to specific circumstances, but to some extent it is present as a consequence of more generic processes of self-organisation operating under physical constraints (Bullock and Buckley, 2009; Bullock et al., 2010; Bartlett and Bullock, 2014). For example, the fact that living systems are embedded in space impacts on the kind of forms that they may readily adopt: not every component of a spatial system may interact directly with every other component; some components are necessarily central, while others must be peripheral, etc.

In this paper we are interested in the impact that these types of constraints have on system structure, and the resultant consequences for system dynamics. We pursue this idea in the context of REDS ${ }^{1}$, a spatially embedded network model that readily exhibits some of the key features of social networks: high clustering, right-skewed degree distribution, positive degree assortativity, and strong community structure (Antonioni et al., 2014).

We first introduce the REDS model and explore how it is affected by the introduction of random rewiring. We demonstrate that rewired REDS networks can be small worlds, i.e., that they exhibit the combination of strong clustering and short characteristic path length that is typical of some social and biological systems. Subsequently, we explore the influence of REDS network structure on the dynamics of two simple contagion processes, one representing the spread of an infectious disease and one representing the invasion of a

\footnotetext{
${ }^{1}$ The REDS acronym relates to the four aspects of a social system that influence network topology within the: the social Reach, Energy, and Synergy parameters of the network, and the Distance between pairs of nodes.
} 
beneficical mutation. We are able to demonstrate that the combination of strong community structure and short path lengths in small world REDS networks facilitates the spread of contagion. The paper closes with a discussion of these results.

\section{Previous Work}

While work on relational (non-spatial) networks dominates network science, there is growing interest in spatially constrained networks (see Barthélemy, 2011, for a recent review of the field). In these models nodes are located in some metric space and connections between nodes are in some way influenced by the distance separating them (see Boguña et al., 2004; Wong et al., 2006; Serrano et al., 2008; zu ErbachSchoenberg et al., 2014, for some recent examples). REDS networks fall within this category and, like many spatial networks, are an extension of Random Geometric Graphs (RGGs), the canonical spatial network model.

A standard RGG can be constructed by distributing $N$ points uniformly at random in some topological space, e.g., the two dimensional unit square, and connecting all pairs of nodes that are separated by a Euclidian distance less than a fixed threshold, $R$. There is an extensive literature on random geometric graphs, particularly in the context of continuum percolation (Dall and Christensen, 2002; Penrose, 2003; Barthélemy, 2011). The degree distribution of a RGG is Poisson with mean equal to $N \pi R^{2}$ (Dall and Christensen, 2002). The clustering coefficient of a RGG tends to $1-\frac{3 \sqrt{3}}{4 \pi} \sim 0.5865$ for all 2-dimensional RGGs in the Euclidean space (Dall and Christensen, 2002), and their assortativity tends to the same value (Antonioni and Tomassini, 2012; Barnett et al., 2007).

RGG networks thus possess some of the properties associated with social networks (strong clustering, positive assortativity, spatiality), but lack others (short characteristic path lengths, strong community structure, long-tailed degree distributions). The REDS model (Antonioni et al., 2014) is an extension to the standard RGG model that is able to readily exhibit all of these properties, save that, until now, REDS networks have not exhibitted the short characteristic path lengths characteristic of small world social networks.

In a REDS network, in addition to the standard RGG constraint that each edge must be shorter than a threshold distance, $R$, all edges impose an energy cost on the nodes that they connect, and the total cost of an individual node's edges may not exceed some finite threshold value, $E$. Each of a node's connections costs an amount of energy proportional to its Euclidean length, $D$. Moreover, the cost of an edge linking two nodes diminishes with the number of neighbours that the two nodes have in common, with the strength of this "synergy" effect governed by a parameter, $S$. Networks are constructed by assigning legal edges at random until no more edges can be afforded.

The resulting REDS networks resemble real social net- works in several respects. They are spatially embedded, have high clustering, positive degree correlation, skewed degree distributions, and strong community structure. However, as mentioned above, standard REDS networks are not small worlds. While they are highly clustered, they have relatively long characteristic path lengths, like lattices. In order to model social processes such as the flow of information or disease in a structured population, it is desirable to employ a random network model that exhibits tuneable analogues of all the major properties of real social networks. Consequently, here we will first explore how readily "small world REDS" can be constructed through a Watts-Strogatzstyle rewiring process (Watts and Strogatz, 1998), before proceeding to explore dynamics on REDS networks for the first time.

\section{Small World REDS Networks}

The REDS model comprises four components:

1. Reach: an undirected edge, $i j$, between a pair of nodes, $i$ and $j$, may only exist if the Euclidean distance between them, $D_{i j}$, is less than their "social reach", $R$.

2. Energy: each node, $i$, has a finite quantity of "social energy", $E$, that may be spent on maintaining its edges.

3. Distance: the cost, $c_{i j}$, of edge $i j$ is proportionate to the Euclidean "social distance", $D_{i j}$, between $i$ and $j$.

4. Synergy: the cost, $c_{i j}$, of edge $i j$ varies inversely with the number of network neighbours that $i$ and $j$ share, $k_{i j}$. This effect is parameterised using $0 \leq S \leq 1^{2}$.

More explicitly, the cost of each edge is calculated as:

$$
c_{i j}=\frac{D_{i j}}{1+S k_{i j}},
$$

where $k_{i j}$, the number of neighbours shared by $i$ and $j$, is the cardinality of the intersection between the set of $i$ 's neighbours and the set of $j$ 's neighbours.

The central intuition of synergy within the REDS model is exemplified as follows. Maintaining relationships with two neighbours that are themselves connected tends to be cheaper than maintaining the same relationships when the two neighbours are not connected to each other. In the former situation, direct interaction with one neighbour effectively involves an element of indirect interaction with the other (through gossip, chance encounters, group gatherings, etc). In more general terms, this is a local network effect that represents the potential for synergetic or catalytic interactions between the system elements.

\footnotetext{
${ }^{2}$ The upper bound on $S$ may appear arbitrary, but $S>1$ would imply that the total cost to a node within a fully connected clique of maintaining its edges would be less than the average distance betweene it and a single neighbour, which we claim to be physcically unrealistic.
} 
Here we construct REDS networks as follows: 1) assign each of $N$ nodes a random location in the unit square, 2) pick a random node, $i, 3)$ pick a random node $j$ such that $D_{i j}<$ $R$ and $j$ is not already a neighbour of $i, 4$ ) if both $i$ and $j$ can afford an edge between them, add it to the network, 5) repeat steps 3) and 4) until no more edges can be added. For a full account of the structural properties of REDS networks, see (Antonioni et al., 2014).

To create small world REDS networks we employ a random rewiring protocol adapted from Watts and Strogatz (1998): for each edge $i j$ in the original network, with probability $p$, remove it and replace it with a new undirected edge $k l$, where $k$ and $l$ are randomly chosen nodes (Newman, 2010, p.555).

\section{Results}

An example REDS network, a rewired REDS network and a RGG with approximately equivalent mean degree are depicted in figure 1 . Note that introducing even a very small amount of random rewiring significantly depresses the characteristic path length of the REDS network, but that it does not impact strongly on the clustering or community structure of the network. Throughout the paper, as a null model against which to compare the results for REDS networks, we will use RGGs with the same number of nodes and a threshold, $R$, that results in approximately equal average degree. Figure 1 shows that such an RGG tends to have less evident community structure, but a somewhat higher average clustering coefficient and also a longer characteristic path length.

Figure 2 depicts a systematic comparison between the effect of random rewiring on REDS networks and RGGs. As the probability of edges being rewired $(p)$ increases, path length falls sharply for both classes of network, whereas clustering falls more slowly, ensuring that a small amount of rewiring can create small world networks where path lengths are relatively low and clustering is relatively high. As $p$ tends to unity, both classes of network converge on fully randomised networks with the same values for clustering and path length.

Figure 3 quantifies the strength of the small world effect, demonstrating that it peaks at similar values of $p$ for both classes of network, with the effect being felt more strongly for RGGs than for REDS networks.

\section{Contagion on REDS Networks}

To explore dynamics on REDS networks, we employ a standard SIS (Susceptible-Infected-Susceptible) model of contagion, a common extension of the classic SusceptibleInfected (SI) model, with the important addition of allowing for recovery and reinfection. It is widely used to model diseases that confer no or limited immunity (Newman, 2010).

Initially, a set of $n$ randomly chosen nodes are set to be infected. The remainder are susceptible. A contagion process

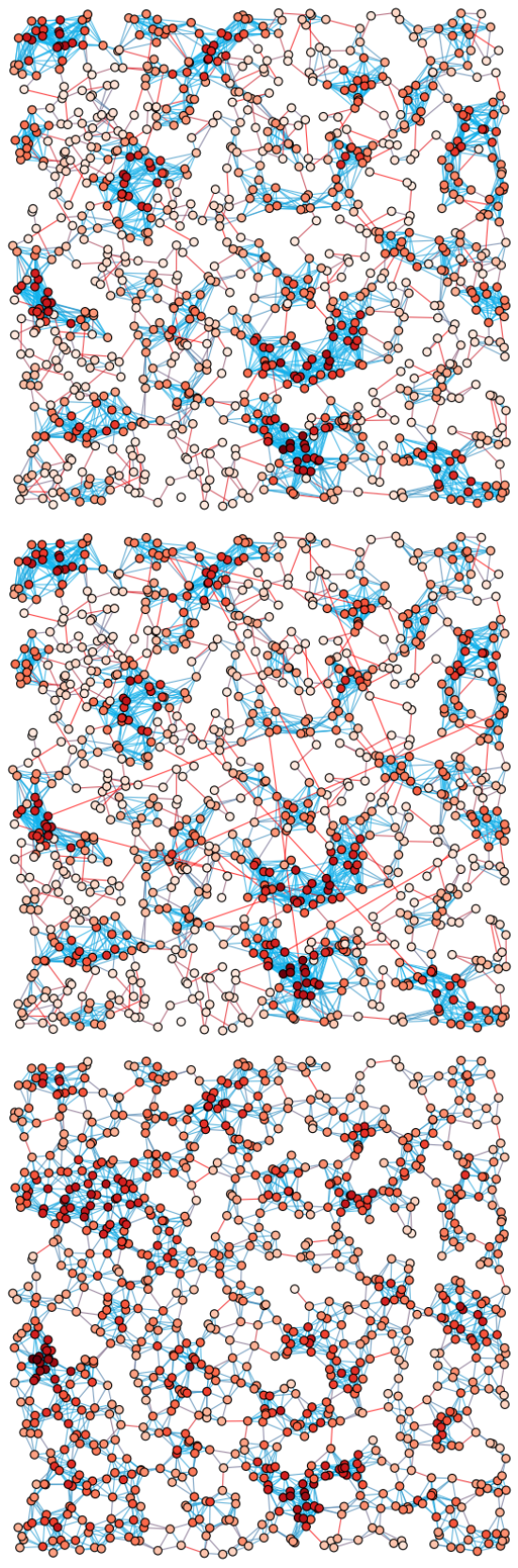

Figure 1: Example networks. Red nodes have higher degree and red edges are more expensive. Top: REDS network $\left(N=10^{3}, R=0.08, E=0.124, S=1.0\right.$, non-toroidal boundaries, mean degree: 8.4 ) exhibiting strong clustering (0.471), evident community structure, and relatively long characteristic path length (13.2). Middle: The same network subject to a small amount of random rewiring ( $p=0.005)$. Path length falls by $\approx 26 \%$ (to 9.71) and clustering by less than $1 \%$ (to 0.467). Bottom: RGG with equivalent degree ( $N=10^{3}, R \approx 0.05$, mean degree: 8.4). Clustering (0.6) and characteristic path length (14.15) are higher than for the REDS network, and respond similarly to random rewiring ( $p=0.005$ causes reductions of $36 \%$ and $2 \%$, respectively). 


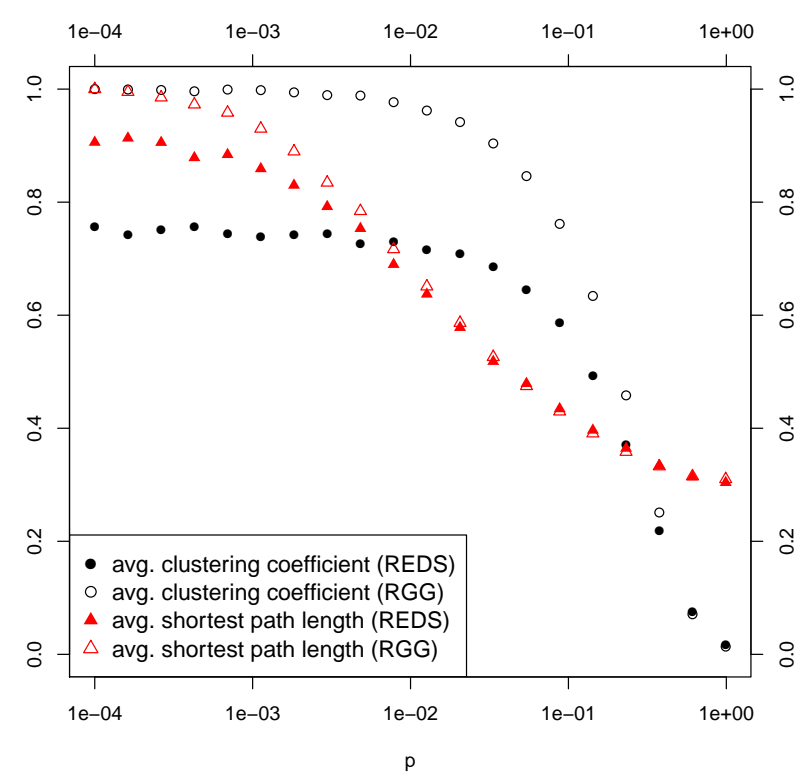

Figure 2: Curves demonstrating the effect of random rewiring on the average clustering coefficients and average shortest (i.e., characteristic) path lengths of REDS networks ( $\left.N=10^{3}, R=0.08, E=0.124, S=1.0\right)$ and RGGs with approximately equivalent degree $\left(N=10^{3}, R=0.05\right)$. Each data point is the average of 100 networks. All values are normalised w.r.t. those of unrewired RGGs. When $p=0$, REDS networks have lower clustering and shorter path lengths than RGGs, but increasing $p$ produces qualitatively equivalent effects on both measures.

then updates the status of each node synchronously for a sequence of discrete time steps during each of which at most one state change is allowed per node. Explicitly, at each time step, $t$ : each node that was susceptible at time step $t-1$ becomes infected with probability $1-(1-\beta)^{\gamma}$, where $\gamma$ is the number of its neighbours that were infected at time step $t-1$ and $0<\beta<1$ is the infection's transmission probability; each node that was infected at time step $t-1$ reverts to being susceptible with probability $\mu$.

Recovery from infection has an important consequence: the number of infected nodes must always be less than the total population. Hence, rather than an outbreak infecting all of the nodes on a network, the system will eventually stabilize when the rates at which new infections arise and infected nodes recover will be exactly equal, resulting in a steady fraction of the population exhibiting the infection (Newman, 2010).

Each finite population exhibits an epidemic threshold, i.e., a ratio between the transmission probability $\beta$ and the recovery probability $\mu$, separating endemic and extinction behaviour. Gomez et al. (2010) proposed a numerical method-

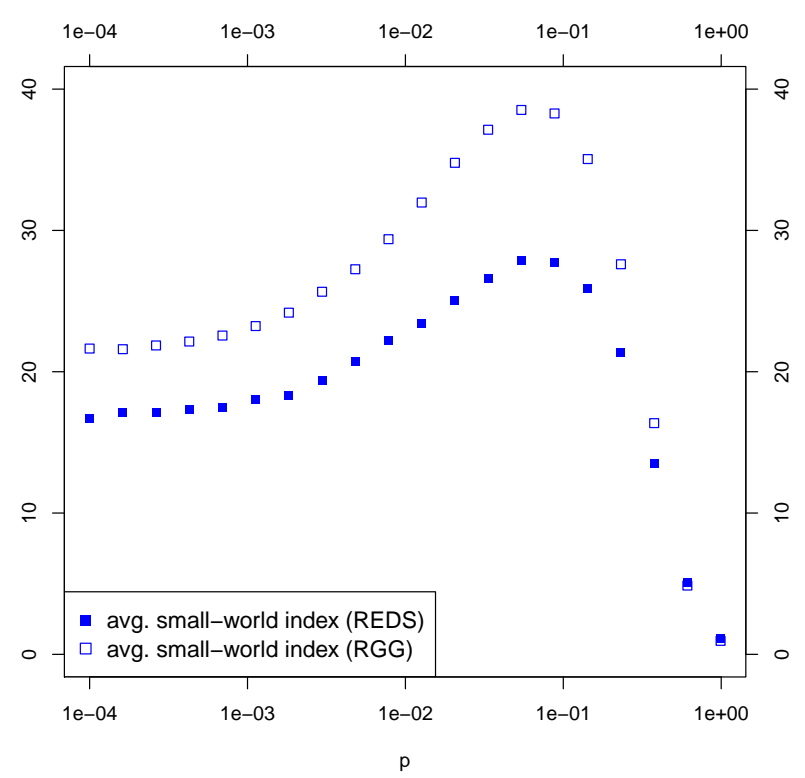

Figure 3: Curves demonstrating the strength of the small world effect brought about by randomly rewiring REDS networks and RGGs (parameters as figure 2). A network's small world index is calculated as $S=\frac{C / C(p=1)}{\lambda / \lambda(p=1)}$, where $C$ and $\lambda$ are the average clustering coefficient and characteristic path length of the network, respectively, while $C(p=1)$ and $\lambda(p=1)$ are the equivalent values calculated for a fully rewired variant of the network. The effect peaks at around the same rewiring probability for both classes of network, but is weaker overall for REDS networks.

ology for calculating this threshold, $\beta_{c}$ (dubbed critical $\beta$ ), based on dividing the recovery probability by the largest eigenvalue of the adjacency matrix $\left(\Lambda_{\max }\right)$ :

$$
\beta_{c}=\frac{\mu}{\Lambda_{\max }}
$$

This provides a quick and easy numerical indicator which, when it coincides with the output of simulations, indicates that the assumptions of the theoretical framework were not violated. Where $\beta<\beta_{c}$, the proportion of infected nodes will go to zero, and we will describe the outbreak as having failed. Where $\beta>\beta_{c}$ the system will stabilize with a positive number of infected nodes and we will describe the outbreak as having reached an endemic state. Moreover, this expression implies that changing the value of $\mu$ only results in a quantitative modification of $\beta_{c}$ without influencing the actual dynamics of the system qualitatively. Therefore, when simulating an epidemic outbreak the value of $\mu$ is typically fixed at unity (Barrat et al., 2008). 


\section{Results}

Here we report the results of SIS processes run on standard REDS networks ( $N=10^{3}, R=0.08, E=0.124, S=1.0$, toroidal boundary conditions), the same REDS networks after suffering some degree of rewiring as described in the previous section, and RGGs that serve as a baseline for comparison, having parameters that give rise to networks with approximately the same average degree $\left(N=10^{3}, R=0.05\right)$.

Due to the heterogeneous nature of our networks, the choice of the initial number of infected nodes can strongly influence the outcome of the simulations. Values reported in the literature range between $0.1 \%$ and $50 \%$ (Ferreri et al., 2014; Pastor-Satorras and Vespignani, 2001). Following Gomez et al. (2010), we chose to infect 5\% (i.e., 50) of the network nodes initially. At lower values, many outbreaks may fail due to topological idiosyncrasies of the randomly chosen initial infected sites.

Each outbreak was simulated using the following protocol:

- Infect $n=50$ unique, randomly selected nodes

- Run the SIS process for a minimum of 300 time steps and a maximum of $10^{4}$ time steps (enough time for the infection to stabilize around its endemic state)

- An infection outbreak is deemed to have stabilized when the median number of infected nodes over the last 100 time steps is between the $49.5^{\text {th }}$ percentile and $50.5^{\text {th }}$ percentile of the previous 100 time steps.

To understand the effect of rewiring on REDS networks, we evaluated trends in $\beta_{c}$, the number of infected nodes after stabilization, and the percentage chance of outbreak failure/survival. Epidemic thresholds observed in simulation agree closely with the expected values.

In the absence of any random rewiring, the $\beta_{c}$ value of a REDS network was determined to be lower than that of an equivalent RGG (figure 4). The net effect of randomly rewiring REDS networks is a monotonic increase in the value of $\beta_{c}$. In other words, the introduction of randomly rewired shortcuts and, more importantly, the resultant progressive loss of an organized community structure reduces the ease with which an outbreak achieves endemic stability. This can be seen in figure 4 and is confirmed numerically in figure 5 .

If we classify this situation in terms of the simple question "does the simulation run survive or die out?", and express the answer as a percentage of total runs, we obtain figure 6 . This classification trades off the ability to separate an endemic state from an epidemic breakout in favour of a clearer separation between the absorbing disease-free regime and the endemic regime, and clearly displays the increased infectiousness required by diseases on networks with higher levels of random rewiring.

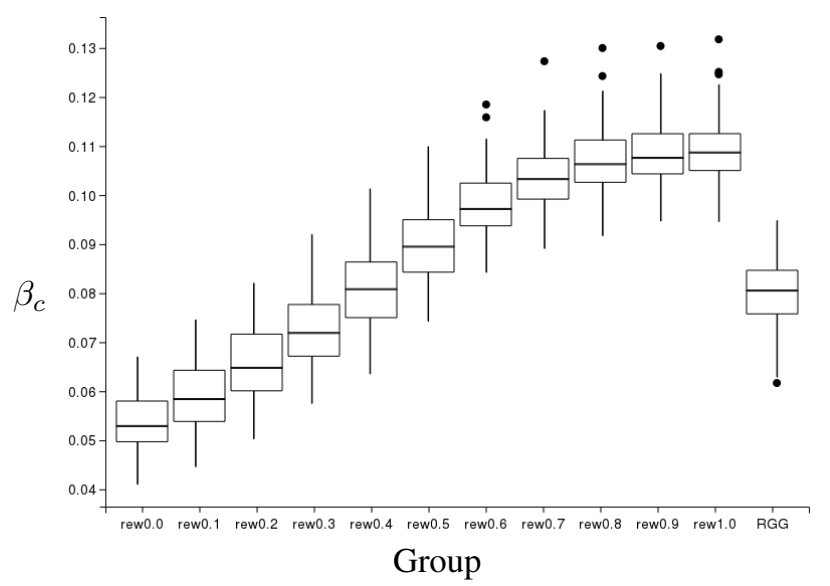

Figure 4: Variation in $\beta_{c}$ for SIS contagion on REDS networks ( $N=10^{3}, R=0.08, E=0.124, S=1.0$ ) subjected to different probabilities of random rewiring. RGGs with approximately equivalent degree $\left(N=10^{3}, R=0.05\right)$ are plotted as a baseline. For all outbreaks, the initial number of infected nodes was $n=50$ and the recovery rate was $\mu=1$. Each box plot represents 100 independent outbreaks simulated on each of 100 networks.

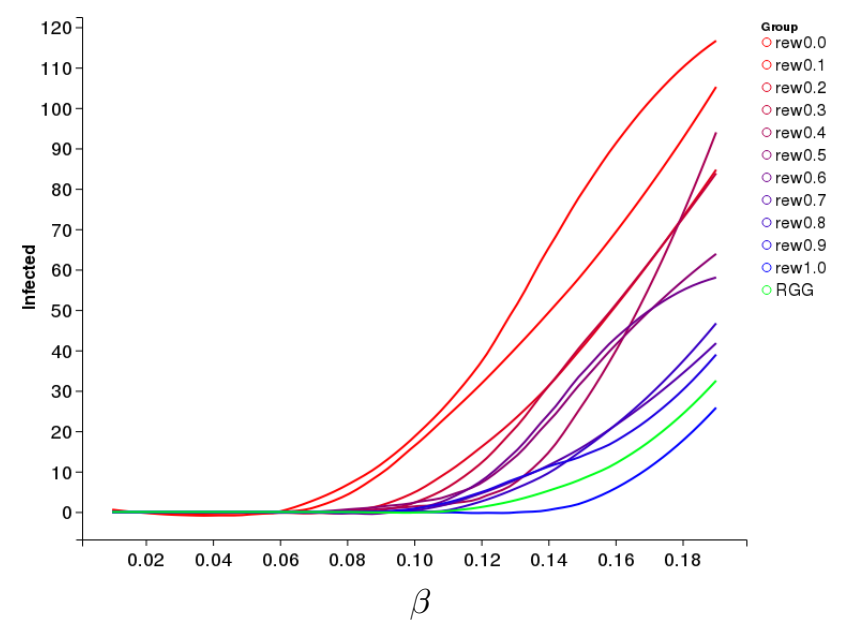

Figure 5: The mean number of infected nodes after stabilization for SIS contagion on REDS networks ( $N=10^{3}, R=$ $0.08, E=0.124, S=1.0)$ subjected to different probabilities of random rewiring. RGGs with approximately equivalent degree $\left(N=10^{3}, R=0.05\right)$ are plotted as a baseline. For all outbreaks, the initial number of infected nodes was $n=50$ and the recovery rate was $\mu=1$. Data plotted represents 100 independent outbreaks simulated on each of 100 networks for each value of $\beta \in[0,0.01,0.02, \ldots]$.

Overall, these results indicate that REDS networks are more susceptible to contagion than equivalent RGGs. Although random rewiring improves their ability to resist infection, a significant amount is required before they are as 


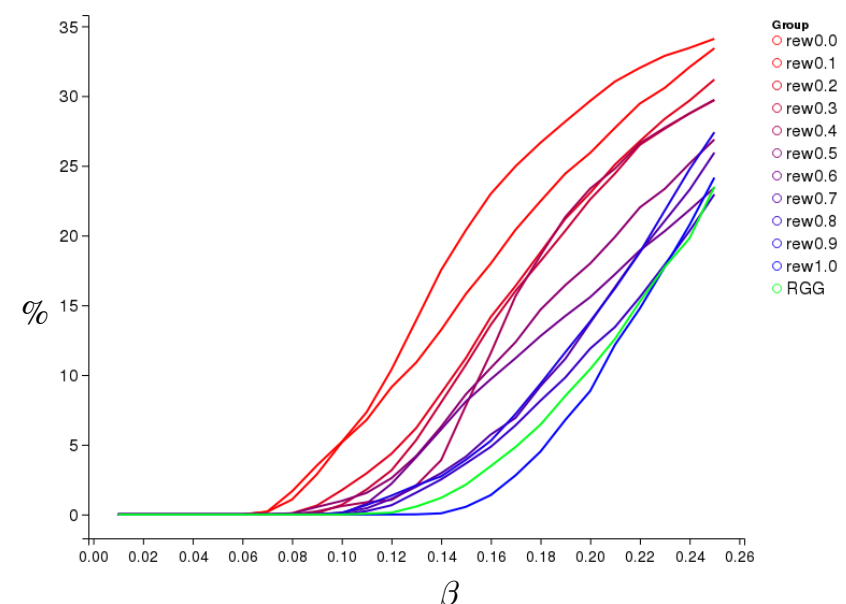

Figure 6: Percentage of SIS outbreaks that do not fail on REDS networks $\left(N=10^{3}, R=0.08, E=0.124, S=\right.$ 1.0) subjected to different probabilities of random rewiring. RGGs with approximately equivalent degree $\left(N=10^{3}\right.$, $R=0.05$ ) are plotted as a baseline. For all outbreaks, the initial number of infected nodes was $n=50$ and the recovery rate was $\mu=1$. Each smoothed curve represents data from 100 independent outbreaks simulated on each of 100 networks for each value of $\beta \in[0,0.01,0.02, \ldots]$.

resistant as equivalent RGGs (e.g., $p \gtrsim 0.4$ in figure 4). Before discussing which properties of REDS networks are responsible for these results, we will explore a contagion dynamic that does not feature recovery and reinfection.

\section{Selection Pressure on REDS Networks}

Whereas the previous section explored a probabilistic contagion process with recovery, we now consider a spreading process without recovery, described in terms of the spread of a beneficial mutant strategy within a population.

When designing an artificial evolutionary system, researchers pay considerable attention to the choice of genetic encoding, parameter values for the genetic operators, and the selection mechanism. Darwin, however, realized that the "populations" that he observed also had spatial structure that influenced their population dynamics. For instance, species isolated on islands evolved differently from their counterparts living in more open environments. Geographical separation is a factor that shapes evolution.

The same principles apply to artificial evolution (Bäck, 1994). In evolutionary systems, topological population structure plays an important role in influencing the dynamical processes taking place. The simultaneous study of the structure and the dynamics of a given system is thus crucial. Structured populations are defined as populations in which any given individual has its own neighborhood, which is generally much smaller than the size of the population (Giacobini et al., 2005, 2004).
Changing the spatial aspect of an evolutionary algorithm influences the selection operator dynamics. In fact, the variation and mutation mechanisms operate on the individuals previously selected, whereas selection is directly influenced by the pool within which each individual is assessed, which is in turn determined by the topology of the population. In the artificial evolutionary environment, the selection mechanism is a key factor in the dynamics of the system, and different selection schemes have been characterized by the selection pressures they induce, usually described in terms of the takeover time. This is defined as being the time it takes for a single best individual to take over the entire population. It can also be seen as a simplified infection process, where an individual is infected when it is replaced by a copy of the best individual and where infected individuals cannot recover but instead stay infected forever. Takeover time can be estimated experimentally by measuring the propagation of the best individual's strategy under the sole effect of selection, without any variation operator. A shorter takeover time indicates a higher selection pressure and thus a more exploitative algorithm. Longer takeover times indicate lower selection pressure and a more exploratory algorithm.

\section{Results}

In order to explore the effect on selection pressure of rewiring REDS networks, we experimentally determine how the takeover time of a single best individual varies with network topology. Again, we explore the full spectrum of rewiring probabilities for REDS networks, $p \in$ $[0,0.01,0.02, \ldots, 1]$, and also for a set of RGGs with approximately equivalent average degree. We generate one hundred different networks for each value of $p$, and also a set of one hundred RGG networks. For each network we repeat the takeover process 10,000 times starting with a randomly chosen initial best individual.

The vertices of each network represent a population of Boolean individuals (where ' 1 ' indicates the presence of the best genotype, and ' 0 ' the presence of some inferior genotype). The edges linking vertices represent the topology over which selection may operate. We analyze the propagation of a single best individual over the entire network using a simple synchronous discrete process that employs a binary tournament for the selection of individuals.

At initialization, all individuals in a population (equivalently: all nodes on the network) are set to ' 0 ' except for a single randomly chosen "best individual" which is set to ' 1 '. Over a finite number of at most 100 time steps, the state of each individual is determined by selecting a single opponent among its neighbors. If the opponent's state is " 1 ", the current individual will adopt that state, otherwise, its state remains unchanged. Thus only the genotype of the best individual propagates through the population. At each time step, we record the fraction of the population with the best genotype. The time step at which this value reaches unity is 


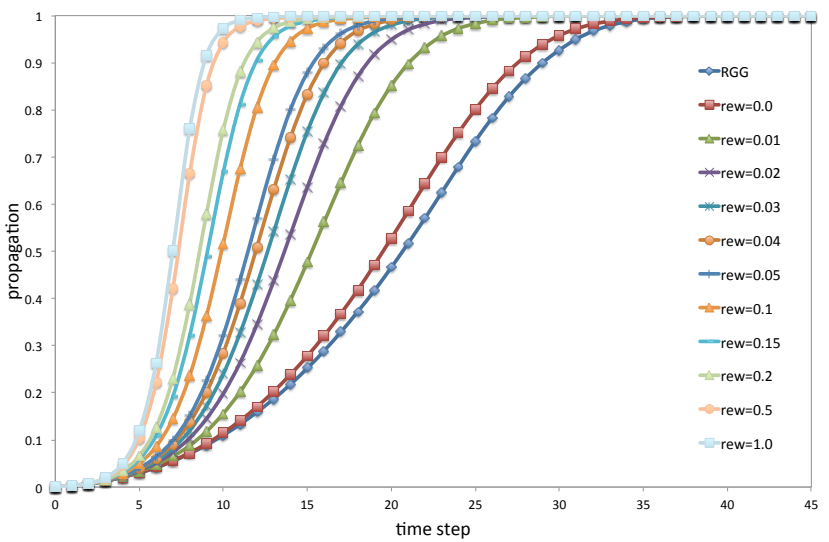

Figure 7: Propagation of the "Best Individual" over REDS networks $\left(N=10^{3}, R=0.08, E=0.124, S=1.0\right)$ subjected to different probabilities of random rewiring, and over RGGs with approximately equivalent degree $\left(N=10^{3}\right.$, $R=0.05$ ).

the takeover time.

Figure 7 represents averaged propagation over time. Each curve is the averaged result of 10,000 independent runs for one hundred networks. To increase the readability of the figure, we have selected a representative subset of rewiring probabilities. Unrewired REDS networks have a slightly faster takeover time than RGGs and hence a slightly higher selection pressure. As the probability of randomly rewiring REDS increases, the selection pressure increases monotonically - rapidly at first and then more slowly. In the next section we will consider why contagion processes on REDS networks respond to rewiring in the way that they do.

\section{Discussion}

We have explored the behaviour of two different contagion processes on REDS networks and on REDS networks that have been subjected to random rewiring. The first (SIS) process involved recovery and reinfection while the second (binary tournament EA) did not.

Before considering the effect of rewiring, we can first observe that REDS networks were more susceptible to contagion of both kinds than equivalent RGGs. For the SIS model, outbreaks were more able to arise and survive on REDS networks than on equivalent RGGs. For the selection pressure simulations, REDS had slightly faster takeover times than equivalent RGGs.

It is known that for diseases from which it is possible to recover and be reinfected (for the SIS results reported here $\mu=1$ means that recovery is automatic) the persistence of the disease in a population is much less likely if that population is well-mixed than if it is more structured (Bolker and Grenfell., 1996). The lack of clustering within a wellmixed population encourages an infection to diffuse rapidly away from the original site of the outbreak and ensures that individuals have little chance of becoming reinfected. Conversely, a population with strong clustering resulting from the presence of local structure ensures that infection spreads to individuals who are able to reinfect one another should they recover, enabling an outbreak to become established in a local portion of the population before spreading.

However, our results suggest that it is not merely the strong clustering in REDS networks that is favouring outbreaks, since the clustering of the equivalent RGGs is higher yet they are more resistant to outbreaks. Rather, it is the stronger community structure of REDS networks that makes them more vulnerable to SIS outbreaks than RGGs. Randomly rewiring REDS networks erodes both clustering and community structure, preventing outbreaks from establishing themselves in a tightly connected local sub-population. However, this effect is gradual, and rewiring must be very prevalent before a REDS network becomes as resistant to outbreaks as an equivalent RGG ( $p$ larger than around 0.4 for the results reported here). Consequently, rewired REDS networks that maximise the small world index (e.g., $p \approx 0.01$ ) are still significantly more vulnerable to disease than equivalent RGGs.

For the second contagion process explored here (takeover of a beneficial mutant on a network via binary tournaments) REDS have an advantage over equivalent RGGs stemming from their shorter characteristic path lengths. Since there is no recovery/reinfection in this spreading process, contagion is not aided by local community structure, but is accelerated by short characteristic path lengths which ensure that one location on the network is only a small number of hops from any other, reducing the diameter of the network.

The increase in selection pressure (i.e., takeover speed) that results from increasing the amount of random rewiring applied to REDS networks is due to the introduction of shortcuts across the networks which shorten their characteristic path lengths and thereby facilitate diffusion.

In summary, on REDS networks a contagion process with recovery/reinfection is inhibited by the introduction of random rewiring, whereas one without recovery/rewiring is $e x$ acerbated by it. Consequently, by comparison with standard RGGs small world REDS networks will be highly susceptible to both kinds of contagion, since they combine strong community structure and relatively short characteristic path lengths.

\section{Conclusion}

In this paper we have explored simple dynamic contagion processes operating over a class of spatially embedded, community structured networks. By introducing a small amount of random rewiring we have been able to demonstrate the existence of a small world regime for REDS networks. We have shown that the strong community structure in REDS networks makes them particularly vulnerable to outbreaks of 
diseases that feature recovery and reinfection. When REDS networks are randomly rewired to the extent that they exhibit a strong small world effect, the consequent erosion of community structure is not enough to protect them from such outbreaks of infection. They remain more vulnerable than standard RGGs. However, since this rewiring does significnatly reduce their characteristic path length, it does accelerate the spread of any contagion that does not feature recovery/reinfection. These results suggest that real-world social networks, i.e., small world networks that are spatially constrained and subject to the influence of some local synergetic network effect, will tend to be more susceptible to a range of different contagion dynamics than was previously thought.

Acknowledgments: Bullock was supported by EPSRC grant EP/H021698/1. Darabos and Moore were supported by NIH grants R01 LM009012 and LM010098. Giacobini acknowledges local research funding from the University of Torino.

\section{References}

Antonioni, A., Bullock, S., and Tomassini, M. (2014). REDS: An energy-constrained spatial social network model. In Lipson, H., Sayama, H., Rieffel, J., Risi, S., and Doursat, R., editors, Proceedings of the Fourteenth International Conference on Artificial Life, pages 368-375. MIT Press, Cambridge MA.

Antonioni, A. and Tomassini, M. (2012). Degree correlations in random geometric graphs. Physical Review E, 86:037101.

Bäck, T. (1994). Selective pressure in evolutionary algorithms: A characterization of selection mechanisms. In Proceedings of the First IEEE Conference on Evolutionary Computation, volume 1, pages 57-62. IEEE Press, Piscataway, NJ.

Barnett, L., Di Paolo, E., and Bullock, S. (2007). Spatially embedded random networks. Physical Review E, 76(5):056115.

Barrat, A., Barthélemy, M., and Vespignani, A. (2008). Dynamical processes on complex networks. Cambridge University Press, Cambridge, UK.

Barthélemy (2011). Spatial networks. Physics Reports, 499:1-101.

Bartlett, S. and Bullock, S. (2014). Natural convection of a two-dimensional Boussinesq fluid does not maximize entropy production. Physical Review E, 90(2):doi:10.1103/PhysRevE.90.023014.

Boerlijst, M. C. and Hogeweg, P. (1991). Spiral wave structures in pre-biotic evolution: Hypercycles stable against parasites. Physica D, 48:17-28.

Boguñá, M., Pastor-Satorras, R., Díaz-Guilera, A., and Arenas, A. (2004). Models of social networks based on social distance attachment. Physical Review E, 70:056122.

Bolker, B. M. and Grenfell., B. T. (1996). Impact of vaccination on the spatial correlation and persistence of measles dynamics. Proceedings of the National Academy of Sciences USA, 93(22):12648-12653.

Buckley, C. L., Bullock, S., and Barnett, L. (2010). Spatially embedded dynamics and complexity. Complexity, 16(2):29-34.
Bullock, S., Barnett, L., and Di Paolo, E. (2010). Spatial embedding and the structure of complex networks. Complexity, 16(2):20-28.

Bullock, S. and Buckley, C. L. (2009). Embracing the tyranny of distance: Space as an enabling constraint. Technoetic Arts, 7(2):141-152.

Dall, J. and Christensen, M. (2002). Random geometric graphs. Physical Review E, 66:016121.

Di Paolo, E. A. (2000). Ecological symmetry breaking can favour the evolution of altruism in an action-response game. Journal of Theoretical Biology, 203:135-152.

Ferreri, L., Bajardi, P., Giacobini, M., Perazzo, S., and Venturino, E. (2014). Interplay of network dynamics and heterogeneity of ties on spreading dynamics. Physical Review E, 90:10.1103/PhysRevE.90.012812.

Giacobini, M., Alba, E., Tettamanzi, A., and Tomassini, M. (2004). Modeling selection intensity for toroidal cellular evolutionary algorithms. In Deb, K., Poli, R., Banzhaf, W., Beyer, H.-G., Burke, E., Darwen, P., Dasgupta, D., Floreano, D., Foster, J., Harman, M., Holland, O., Lanzi, P., Spector, L., Tettamanzi, A., Thierens, D., and Tyrrell, A., editors, Genetic and Evolutionary Computation - GECCO 2004, pages 1138-1149. Springer, Berlin Heidelberg.

Giacobini, M., Tomassini, M., Tettamanzi, A., and Alba, E. (2005). Selection intensity in cellular evolutionary algorithms for regular lattices. IEEE Transactions on Evolutionary Computation, 9(5):489-505.

Gomez, S., Arenas, A., Borge-Holthoefer, J., Meloni, S., and Moreno., Y. (2010). Discrete-time markov chain approach to contact-based disease spreading in complex networks. $E \mathbf{E}$ rophysics Letters, 89:38009.

Newman, M. E. J. (2010). Networks: An Introduction. Oxford University Press, Oxford.

Pastor-Satorras, R. and Vespignani, A. (2001). Epidemic dynamics and endemic states in complex networks. Physical Review E, 63(6):066117.

Penrose, M. (2003). Random Geometric Graphs. Oxford University Press, Oxford, UK.

Serrano, M., Krioukov, D., and Boguná, M. (2008). Self-similarity of complex networks and hidden metric spaces. Physical Review Letters, 100:078701.

Watts, D. J. and Strogatz, S. H. (1998). Collective dynamics of 'small-world' networks. Nature, 393:440-442.

Wong, L. H., Pattison, P., and Robins, G. (2006). A spatial model for social networks. Physica A, 360(1):99-120.

zu Erbach-Schoenberg, E., Bullock, S., and Brailsford, S. (2014). A model of spatially constrained social network dynamics. Social Science Computer Review. 0894439313511934. 